\title{
Higgs production in neutralino decays in the MSSM
}

\author{
The $\mathrm{LHC}$ and a future $e^{+} e^{-}$collider
}

\author{
A. Arbey ${ }^{1,2,3}$, M. Battagliaa ${ }^{3,4, a}$, F. Mahmoudi ${ }^{1,2,3}$ \\ ${ }^{1}$ Université Lyon 1, Centre de Recherche Astrophysique de Lyon, CNRS, UMR 5574, 69561 Saint-Genis Laval Cedex, France \\ ${ }^{2}$ Ecole Normale Supérieure de Lyon, Lyon, France \\ ${ }^{3}$ CERN, 1211 Geneva 23, Switzerland \\ ${ }^{4}$ Santa Cruz Institute of Particle Physics, University of California, Santa Cruz, CA 95064, USA
}

Received: 15 December 2014 / Accepted: 10 February 2015 / Published online: 6 March 2015

(C) The Author(s) 2015. This article is published with open access at Springerlink.com

\begin{abstract}
The search for the production of weakly interacting SUSY particles at the LHC is crucial for testing supersymmetry in relation to dark matter. Decays of neutralinos into Higgs bosons occur over some significant part of the SUSY parameter space and represent the most important source of $h$ boson production in SUSY decay chains in the MSSM. We study $h$ production in neutralino decays using scans of the phenomenological MSSM. Whilst in constrained MSSM scenarios the decay $\tilde{\chi}_{2}^{0} \rightarrow h \tilde{\chi}_{1}^{0}$ is the dominant channel, this does not hold in more general MSSM scenarios. On the other hand, the $\tilde{\chi}_{2,3}^{0} \rightarrow h \tilde{\chi}_{1}^{0}$ decays remain important and are highly complementary to multi-lepton final states in the LHC searches. The perspectives for the LHC analyses at 8 and $14 \mathrm{TeV}$ as well as the reach of an $e^{+} e^{-}$collider with $\sqrt{s}=0.5,1,1.5$ and $3 \mathrm{TeV}$ are discussed.
\end{abstract}

\section{Introduction}

The discovery of a Higgs boson by the ATLAS and CMS experiments $[1,2]$ at the CERN LHC has opened an era of detailed studies of its production and decay properties. In particular, establishing if the discovered particle is the Standard Model (SM) Higgs boson or the manifestation of an extended Higgs sector is a key question. If the observed Higgs particle is the lightest Higgs boson, $h$, of a supersymmetric extension of the SM (SUSY), it could be significantly produced also in the decay chains of supersymmetric particles. We shall show here that the decays of neutralinos, $\tilde{\chi}_{j}^{0} \rightarrow \tilde{\chi}_{i}^{0} h$ are the most important process

a e-mail: MBattaglia@1bl.gov leading to the production of a $h$ boson in a SUSY decay chain in the MSSM. There have been several phenomenological studies of $h$ production in neutralino decays in various constrained supersymmetric models [3-11], including some detailed assessments of the LHC potential [7, 12-14]. Results from searches for neutralino and chargino production conducted by ATLAS and CMS at the LHC have been reported for the two [15-18] and three [16,18-20] lepton channels as well as the $b b \ell+$ MET channel [21,22], sensitive to $h$ production in neutralino decays. This paper discusses the regions of the MSSM parameters where these decays into the lightest Higgs boson are relevant and the perspectives for their search at the LHC and a future lepton collider in the framework of the phenomenological minimal supersymmetric extension of the SM (pMSSM) with 19 free parameters [23]. This framework provides sufficient freedom to the masses and couplings to explore the supersymmetric parameter space in a largely unbiased way and has been adopted in phenomenological [24-29] and experimental [30,31] studies. The study of the pMSSM parameter space with high statistics, flat scans of its parameters is briefly described in Sect. 2. Results from measurements and searches at LEP and LEP2, flavour physics and dark matter experiments already provide powerful constraints. These are discussed in Sect. 3 together with the implications of the LHC results from the search for SUSY particles and the first determination of the properties of the discovered boson. Section 4 presents the regions of the viable pMSSM parameter space probed by the different channels of neutralino decays, in particular those involving the lightest Higgs bosons. In Sect. 5, we contrast the results for the pMSSM with those obtained with the highly constrained, 5-parameter CMSSM [32,33], which have been widely studied in relation to dark matter [3439] and for benchmarking [40-42]. Section 6 discusses the perspectives for the searches at LHC and a lepton collider, 
for various centre-of-mass energies, while Sect. 7 has the conclusions.

\section{MSSM scans and tools}

This study is based on the analysis of a large sample of MSSM points obtained through a flat scan of the pMSSM parameters, which are varied in an uncorrelated way within the following ranges:

$$
\begin{aligned}
& 1 \leq \tan \beta \leq 60, \\
& 50 \mathrm{GeV} \leq M_{A} \leq 2 \mathrm{TeV}, \\
& -10 \mathrm{TeV} \leq A_{f} \leq 10 \mathrm{TeV}, \\
& 50 \mathrm{GeV} \leq M_{\tilde{f}_{L}}, M_{\tilde{f}_{R}}, M_{3} \leq 3.5 \mathrm{TeV}, \\
& 50 \mathrm{GeV} \leq M_{1}, M_{2},|\mu| \leq 3.0 \mathrm{TeV}
\end{aligned}
$$

to generate a total of $2 \times 10^{8} \mathrm{pMSSM}$ points. We also perform a scan of the CMSSM, in order to contrast the results for the pMSSM with those of a highly constrained model. For this we vary the CMSSM parameters within the following ranges:

$$
\begin{aligned}
& 1 \leq \tan \beta \leq 60 \\
& -10 \mathrm{TeV} \leq A_{0} \leq 10 \mathrm{TeV} \\
& 0 \leq M_{0}, M_{1 / 2} \leq 3.5 \mathrm{TeV}
\end{aligned}
$$

In all scans, we select the generated points which fulfil a set of constraints derived from flavour physics and lower energy searches at LEP2 and the Tevatron, as summarised below and discussed in more details in Ref. [26], to which we refer also for further details on the pMSSM scans. These selected points are referred to as "accepted points" in the following. The pMSSM scans employ a number of programs and software tools. Only those most relevant to this study, are mentioned here, while further details are given in [26]. SUSY mass spectra are generated with SOFTSUSY 3. 2 . 3 [43]. Decay branching fractions are calculated using SDECAY $[44,45]$. Higgs decay branching fractions are calculated with HDECAY V5. 0 [46]. The flavour observables and dark matter relic density are calculated with Super Iso Relic v3.2 [47-49]. Cross sections are computed with Pythia 6.424 [50], also used for event generation. The NLOk-factors are evaluated using Prospino v2 [51]. The physics object response for the LHC analyses is simulated using the Delphes 3.0 fast simulation package $[52,53]$, tuned on the ATLAS detector performance and validated for the ATLAS multi-lepton neutralino and chargino analyses.

\section{Constraints}

We apply constraints from flavour physics, dark matter and SUSY searches at LEP, Tevatron and LHC. These have been discussed in details in Ref. [26]. In particular, we consider the decay $B_{S} \rightarrow \mu^{+} \mu^{-}$, which can receive significant SUSY contributions at large values of $\tan \beta$. Here, we adopt the latest combined results from LHCb and CMS with the measurement of a branching fraction of $\operatorname{BR}\left(B_{s}^{0} \rightarrow \mu^{+} \mu^{-}\right)=$ $(2.8 \pm 0.7) \times 10^{-9}[54-56]$ from which we derive the constraints at $95 \%$ C.L. after accounting for theoretical uncertainties. Dark matter also provides significant bounds for this analysis. We impose the constraint on the neutralino relic density of $10^{-4}<\Omega_{\chi} h^{2}<0.163$, derived from the PLANCK satellite result [57], accounting for theoretical and cosmological uncertainties and allowing the neutralino be responsible for only part of the observed dark matter. In addition, we compare the sensitivity of the LHC searches to the new limits on dark matter direct detection for spin-independent $\tilde{\chi}$-nucleon scattering obtained from the XENON-100 [58] and the LUX [59] data.

Moreover, we apply the constraints in the Higgs sector by selecting only points having a mass of the lightest Higgs boson, $h$, in the range $123<M_{h}<129 \mathrm{GeV}$ and compatible with the constraints on the heavier MSSM Higgs bosons in the channel $H / A \rightarrow \tau^{+} \tau^{-}$[62].

Searches for SUSY particles at the LHC in channels with missing $E_{T}$ have already provided a number of constraints relevant to this study, by excluding part of the pMSSM phase space, corresponding to masses of the gluino and scalar quarks of the first two generations below $\sim 600 \mathrm{GeV}$ to $1 \mathrm{TeV}$ and those of scalar bottom and top below $\sim 400$ to $800 \mathrm{GeV}$. Here, we use these results to compare the sensitivity of electroweak SUSY particle production to that of the ATLAS searches for scalar quarks of the first two generations and gluinos in the jets + MET channel [60] and for scalar top and bottom quarks with $b$-tagged jets + MET [61].

\section{Neutralino decays to $h$ in the MSSM}

Neutralino decays are the most important source of $h$ bosons in SUSY decays in the MSSM. The study of $h$ bosons appearing in the decay of SUSY particles in models obtained by scanning the pMSSM parameter space shows that in $53 \%$ of the cases they originate from neutralino decays, either from direct production or from a scalar quark decay chain. This value slightly exceeds that obtained for the occurrence of chargino decays $\tilde{\chi}_{2}^{ \pm} \rightarrow h \tilde{\chi}_{1}^{ \pm}(45 \%)$ while the $\tilde{\tau}_{2}^{ \pm} \rightarrow h \tilde{\tau}_{1}^{ \pm}$, $\tilde{t}_{2} \rightarrow h \tilde{t}_{1}$ and $\tilde{b}_{2} \rightarrow h \tilde{b}_{1}$ processes have only a marginal contribution (2\%). The occurrence of $h$ bosons in SUSY decays characterises $\sim 1.5 \%$ of the accepted pMSSM points considered in this study. In the MSSM the couplings of the Higgs bosons to charginos and neutralinos are maximal for higgsino-gaugino mixed states, while the gauge boson couplings to neutralinos are maximal for higgsino-like states. 

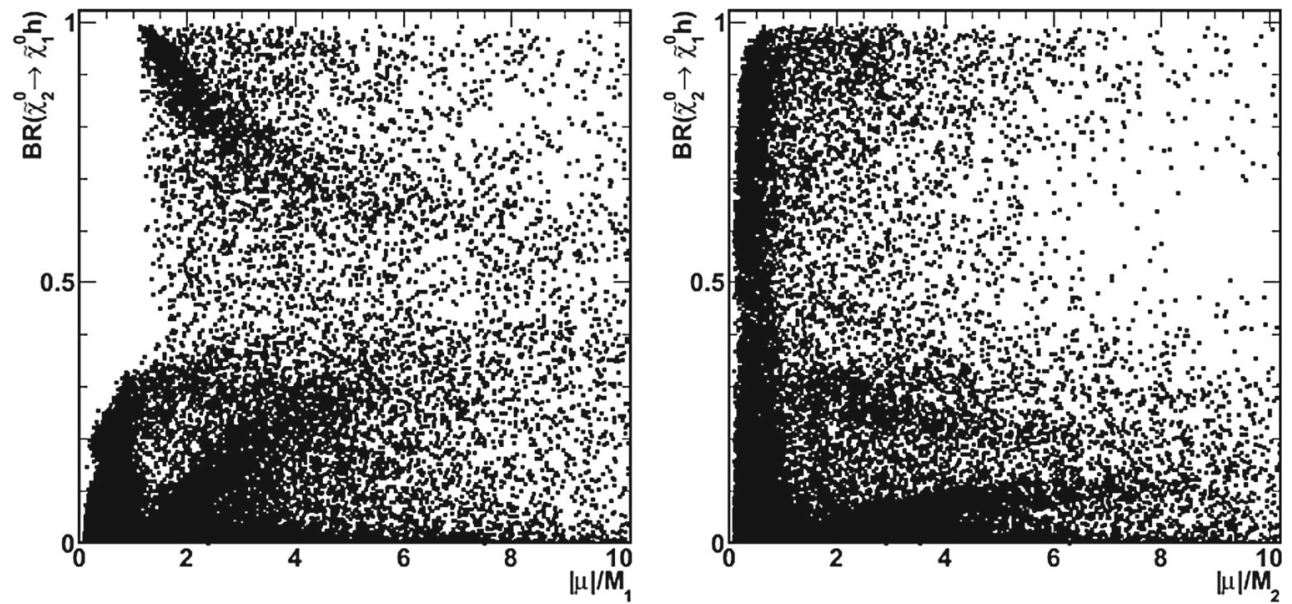

Fig. 1 The branching fraction $\tilde{\chi}_{2}^{0} \rightarrow h \tilde{\chi}_{1}^{0}$ for accepted pMSSM points as a function of the ratio $|\mu| / M_{1}($ left $)$ and $|\mu| / M_{2}$ (right). The upper-left branches in both plots are due to bino-like $\tilde{\chi}_{2}^{0}$
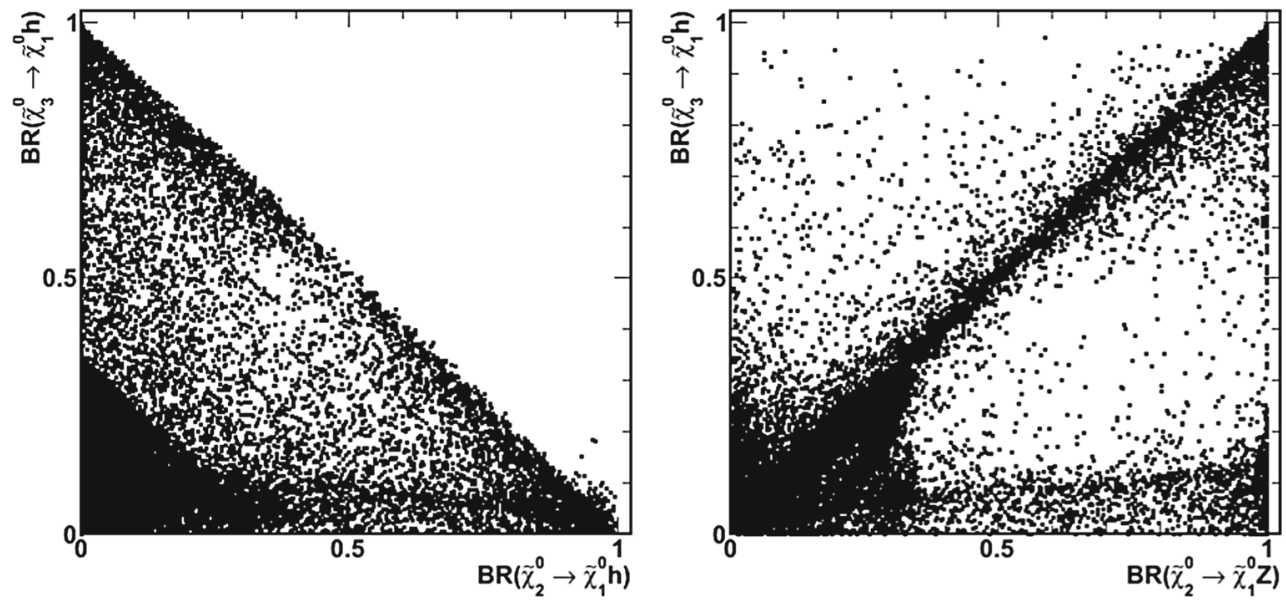

Fig. 2 The branching fraction $\tilde{\chi}_{3}^{0} \rightarrow h \tilde{\chi}_{1}^{0}$ as a function of that for $\tilde{\chi}_{2}^{0} \rightarrow h \tilde{\chi}_{1}^{0}($ left $)$ and $\tilde{\chi}_{3}^{0} \rightarrow h \tilde{\chi}_{1}^{0}$ as a function of that for $\tilde{\chi}_{2}^{0} \rightarrow Z \tilde{\chi}_{1}^{0}($ right $)$ for accepted pMSSM points, showing the complementarity of the decays of $\tilde{\chi}_{3}^{0}$ and $\tilde{\chi}_{2}^{0}$ to $h$ and $Z$

The $h$ couplings to neutralino are suppressed by powers of $|\mu| / M_{2}$ or $M_{2} /|\mu|$ in the gaugino-like and higgsino-like regions, respectively. The branching fraction of $\tilde{\chi}_{2}^{0} \rightarrow h \tilde{\chi}_{1}^{0}$ for a set of accepted pMSSM points is shown as a function of $|\mu| / M_{1}$ and $|\mu| / M_{2}$ in Fig. 1. The larger values of this branching fraction are obtained mostly in the region where $|\mu| \lesssim M_{2}$ and the neutralino is bino. On the contrary, in the region where $|\mu| \lesssim M_{1}$ the branching ratio is limited to no more than $30 \%$.

In the case the charginos and neutralinos are gaugino-like (i.e. when the higgsino mass parameter is much larger than the wino mass parameter, $|\mu| \gg M_{2}$ ) or higgsino-like (i.e. in the opposite situation $|\mu| \ll M_{2}$ ), this results in the dominance of the decays of the heavier charginos and neutralinos into the lighter states and Higgs bosons, over the same decays with gauge boson final states [6,7]. This is also the case for the so-called "little cascade" in the region $M_{2} \gtrsim M_{1} \gg|\mu|$ where the branching fraction for the decay of the $\tilde{\chi}_{2}^{0}$ into the LSP neutralino and the lighter $h$ boson $\tilde{\chi}_{2}^{0} \rightarrow h \tilde{\chi}_{1}^{0}$ is in general larger than that for the decay $\tilde{\chi}_{2}^{0} \rightarrow Z \tilde{\chi}_{1}^{0}$, when kinematically accessible in the two-body channel. ${ }^{1}$ Thus, the decay $\tilde{\chi}_{2}^{0} \rightarrow h \tilde{\chi}_{1}^{0}$ is important in these regions, unless sleptons are light and the additional channels where $\tilde{\chi}_{2}^{0}$ decays into a lepton and its super-partner are open. These decays are most important if the neutralino is gaugino-like, since the coupling to the higgsino component is suppressed due to the small lepton mass.

It is important to observe that for small values of ||$\mu \mid-$ $M_{2}$ |, where the contribution of the decay into $h$ is enhanced, the mass difference $M_{\chi_{3}^{0}}-M_{\chi_{2}^{0}}$ is small, typically of the order of $50 \mathrm{GeV}$ or less. In this regime, the production cross sections for $\tilde{\chi}_{2}^{0} \tilde{\chi}_{1}^{ \pm}$and $\tilde{\chi}_{3}^{0} \tilde{\chi}_{1}^{ \pm}$in $p p$ collisions are comparable. Due to the nature of the $\tilde{\chi}_{2}^{0}$ and $\tilde{\chi}_{3}^{0}$ states, the branching

\footnotetext{
${ }^{1}$ In the three-body process, the $h$ has to be virtual and the rate is suppressed by the small couplings of the $h$ state to light fermions.
} 
fractions into $h$ and $Z$ of these two neutralinos are complementary, i.e. the yield into $h$ for the $\tilde{\chi}_{2}^{0}$ is approximately equal to that into $Z$ of the $\tilde{\chi}_{3}^{0}$ states and vice versa. This is illustrated in Fig. 2 which shows the branching fraction of $\tilde{\chi}_{3}^{0} \rightarrow h \tilde{\chi}_{1}^{0}$ as a function of those for $\tilde{\chi}_{2}^{0} \rightarrow h \tilde{\chi}_{1}^{0}$ and $\tilde{\chi}_{2}^{0} \rightarrow Z \tilde{\chi}_{1}^{0}$. The left panel reveals the anti-correlation between the $\tilde{\chi}_{2}^{0}$ and $\tilde{\chi}_{3}^{0}$ decays, while that on the right shows the linear correlation between $\tilde{\chi}_{3}^{0} \rightarrow h \tilde{\chi}_{1}^{0}$ and $\tilde{\chi}_{2}^{0} \rightarrow Z \tilde{\chi}_{1}^{0}$ decays for most of the accepted points. This highlights the complementarity between the search for $\tilde{\chi}_{2}^{0}$ and $\tilde{\chi}_{3}^{0}$ with decays into $h$ and $Z$ and the interest in pursuing both in the LHC analyses.

We study the regions explorable by the $\tilde{\chi}_{2,3}^{0} \rightarrow h \tilde{\chi}_{1}^{0}$ decay using our set of accepted pMSSM points. The extension of these regions depends only on a subset of the pMSSM parameters, mostly $M_{1}, M_{2}, \mu, M_{2}-M\left(\tilde{e}_{L, R}\right)$. However, the constraints discussed in the previous section introduce non-trivial correlations with the other pMSSM parameters and make advantageous to perform this study by keeping all the parameters free. In order to characterise the occurrence of the various decays in different regions of the parameter space, we first study the fraction of the accepted pMSSM points into each bin in the $\left[\mu, M_{1}\right]$ and $\left[\mu, M_{2}\right]$ planes where $h \tilde{\chi}_{1}^{0}, Z \tilde{\chi}_{1}^{0}$ and the sum of $\tilde{\ell} \ell$ and $\tilde{\tau} \tau$ is the dominant two-body neutralino decay mode. We define the dominant decay mode as that having a branching fraction larger than both 0.2 and any of the other two-body decay channels. Results are shown for $\tilde{\chi}_{2}^{0}$ in Figs. 3 and 4, where we also show the region where the sum of branching fractions for the three-body decays exceeds both 0.2 and any of the individual two-body processes. While the exact values for the fraction of points depend on the parameter ranges adopted for our scans, given in Eq. (1), the location of the parameter regions yielding decays into $h$ bosons from Fig. 4 is of general validity.

As shown in Fig. 3 , the $h \tilde{\chi}_{2}^{0} \tilde{\chi}_{1}^{0}$ couplings are larger than the $Z \tilde{\chi}_{2}^{0} \tilde{\chi}_{1}^{0}$ whenever $M_{1} \lesssim|\mu|$ and the $\tilde{\chi}_{1}^{0}$ is mostly bino. The region where $|\mu| \approx M_{1}$ is excluded as the decay $\tilde{\chi}_{2}^{0} \rightarrow h \tilde{\chi}_{1}^{0}$ is not kinematically allowed. Very small values of $|\mu|$ are excluded from the searches for charginos at LEP2, which implies that $|\mu| \gtrsim 90 \mathrm{GeV}$. The $\tilde{\chi}_{2}^{0} \rightarrow Z \tilde{\chi}_{1}^{0}$ mode is dominant for low values of $\mu$ where the LSP is higgsino-like and, thus, there is a strong coupling of the $Z$ boson to $\tilde{\chi}_{2}^{0} \tilde{\chi}_{1}^{0}$ states. There is a line $M_{Z} \lesssim M_{\chi_{2}^{0}}-M_{\chi_{1}^{0}} \lesssim M_{h}$ in which the decay channel $\tilde{\chi}_{2}^{0} \rightarrow h \tilde{\chi}_{1}^{0}$ is kinematically closed and the decay $\tilde{\chi}_{2}^{0} \rightarrow Z \tilde{\chi}_{1}^{0}$ becomes dominant, provided that the sleptons are not light enough for the $\tilde{\ell} \ell$ channel to contribute. Finally, at small $|\mu|$ values, $\tilde{\chi}_{1}^{0}$ and $\tilde{\chi}_{2}^{0}$ are higgsino-like, but the splitting is typically small, so the two-body decays through $h$ or $Z$ are forbidden and the three-body modes dominate. It is important to point out that the parameters which increase $\tilde{\chi}_{2,3}^{0} \rightarrow h \tilde{\chi}_{1}^{0}$ do not cause an increase of the $h$ decays into $\tilde{\chi}_{1}^{0} \tilde{\chi}_{1}^{0}$ pairs and the branching fractions for the two processes are largely uncorrelated. Therefore, the rejection of
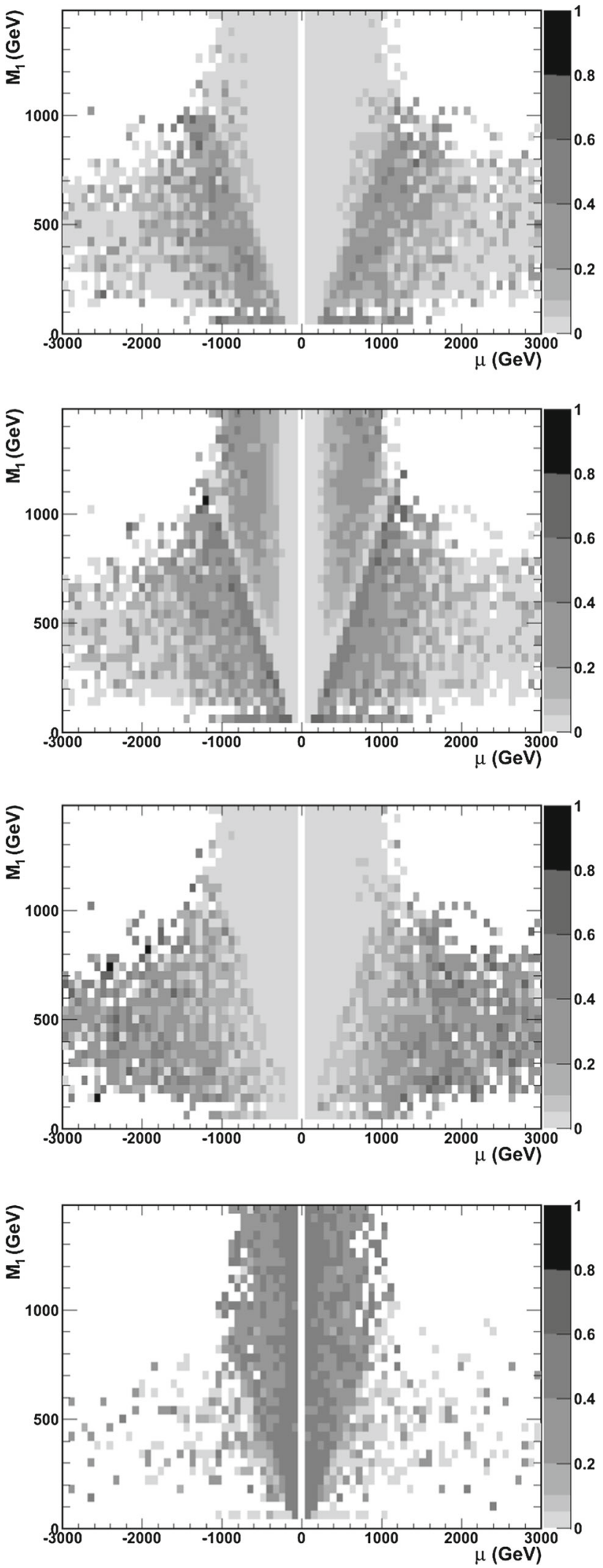

Fig. 3 Fraction of accepted pMSSM points in the $\left[\mu, M_{1}\right]$ MSSM plane having $\tilde{\chi}_{2}^{0} \rightarrow h \tilde{\chi}_{1}^{0}$ (top) $Z$ (upper), $\tilde{\ell} \ell$ or $\tilde{\tau} \tau$ (centre) and threebody (lower) as dominant decay 

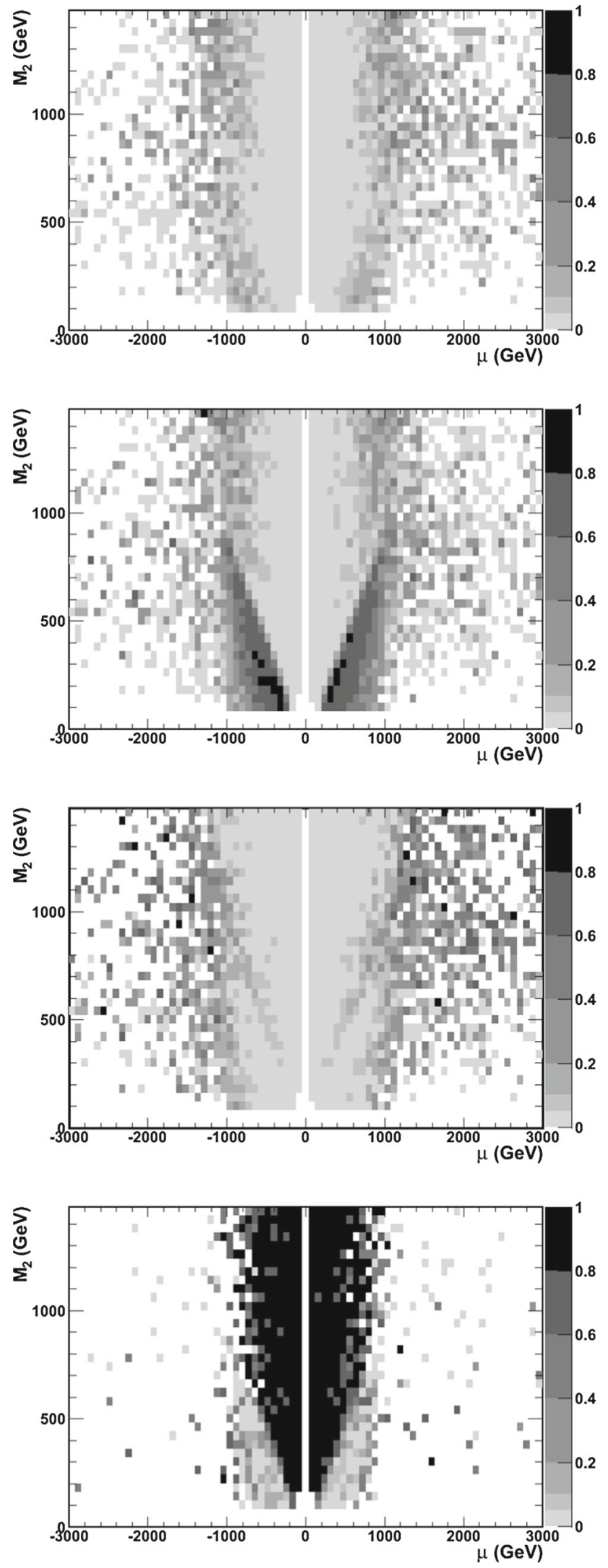

Fig. 4 Same as Fig. 3 for the $\left[\mu, M_{2}\right]$ MSSM plane with the fraction of accepted pMSSM points having $\tilde{\chi}_{2}^{0} \rightarrow h \tilde{\chi}_{1}^{0}$ (top) $Z$ (upper), $\tilde{\ell} \ell$ or $\tilde{\tau} \tau$ (lower) and three-body (bottom) as dominant decay the pMSSM points giving invisible Higgs decay rates above $15 \%$ does not reduce the occurrence of neutralino decays into the lightest Higgs boson. The dependence of the dominant channel on $M_{2}$ is less pronounced, as shown in Fig. 4. In the region where $M_{2} \lesssim|\mu|$, the $\tilde{\chi}_{2}^{0} \rightarrow Z \tilde{\chi}_{1}^{0}$ is dominant, while the three-body channels become very important for $|\mu| \lesssim M_{2}$ due to the small mass splitting between the two light higgsinos.

Through the decays $h \rightarrow b \bar{b}, h \rightarrow \tau^{+} \tau^{-}, Z \rightarrow b \bar{b}$, $Z \rightarrow \tau^{+} \tau^{-}$, these processes lead to the final states $b \bar{b} \ell$ $+E_{T}^{\text {missing }}$ and $\tau \tau \ell+E_{T}^{\text {missing }}$ at the LHC, where the $\tilde{\chi}_{2,3}^{0}$ is produced in association with a chargino decaying $\tilde{\chi}_{1}^{ \pm} \rightarrow W^{ \pm} \tilde{\chi}_{1}^{0} \rightarrow \ell^{ \pm} \nu \tilde{\chi}_{1}^{0}$. An alternative, intriguing scenario leading to the same $\tau \tau \ell+E_{T}^{\text {missing }}$ final state arises if the $\tilde{\tau}_{1}$ is light. In this case, the decay $\tilde{\chi}_{2}^{0} \rightarrow \tilde{\tau}_{1} \tau \rightarrow \tau \tau \tilde{\chi}_{1}^{0}$ may be enhanced.

\section{Neutralino decays to $h$ : pMSSM to CMSSM comparison}

$W h$ production from $\tilde{\chi}_{1}^{ \pm} \tilde{\chi}_{2}^{0}$ decays at the LHC has been discussed in the context of the CMSSM in Ref. [63], where it is found that the decay $\tilde{\chi}_{2}^{0} \rightarrow h \tilde{\chi}_{1}^{0}$ is dominant for typical choices of the model parameters. In our analysis we find indeed that $\tilde{\chi}_{2}^{0} \rightarrow h \tilde{\chi}_{1}^{0}$ is the dominant decay, with a branching fraction in excess of 0.20 , for $71 \%$ of the accepted CMSSM points before applying the relic density constraint. However, this is no longer the case when we impose the full set of constraints. Of the accepted points with $\tilde{\chi}_{2}^{0} \rightarrow h \tilde{\chi}_{1}^{0}$ as the dominant decay, only $10^{-4}$ fulfil the constraint $10^{-4}<\Omega_{\chi} h^{2}<0.163$ and $8 \times 10^{-5}$ the tighter requirement of $0.076<\Omega_{\chi} h^{2}<0.163$ set by the PLANCK cosmic microwave background (CMB) bound. In fact, the region where $\tilde{\chi}_{2}^{0} \rightarrow h \tilde{\chi}_{1}^{0}$ is dominant corresponds to values of neutralino relic density, $\Omega_{\chi} h^{2}$, which largely exceed the upper limit of the PLANCK result [57], as shown in Fig. 5. This is due to the peculiar nature of the CMSSM, which has large $\mu$ values, with $|\mu| \gg M_{2}$, making the neutralinos gaugino-like, as observed above. The general prevalence of the $\tilde{\chi}_{2}^{0} \rightarrow h \tilde{\chi}_{1}^{0}$ decay, observed in the CMSSM before the $\Omega_{\chi} h^{2}$ constraint, cannot be generalised to the MSSM. But in turn the greater flexibility of the pMSSM allows one to reconcile the relic density constraint to large coupling of the $h$ to the neutralinos (see Fig. 5).

For comparison, in the pMSSM the decay into $\tilde{\chi}_{1}^{0} h$ is dominant in only $10 \%$ of the accepted points before the relic density constraint. But now $28 \%$ of these fulfil also the loose relic density constraint of $10^{-4}<\Omega_{\chi} h^{2}<0.163$ and still $2.2 \%$ make also the tighter requirement of $0.076<\Omega_{\chi} h^{2}<$ 0.163 . These points are almost all in the so-called " $A^{0}$ annihilation funnel", where neutralinos in the early universe anni- 

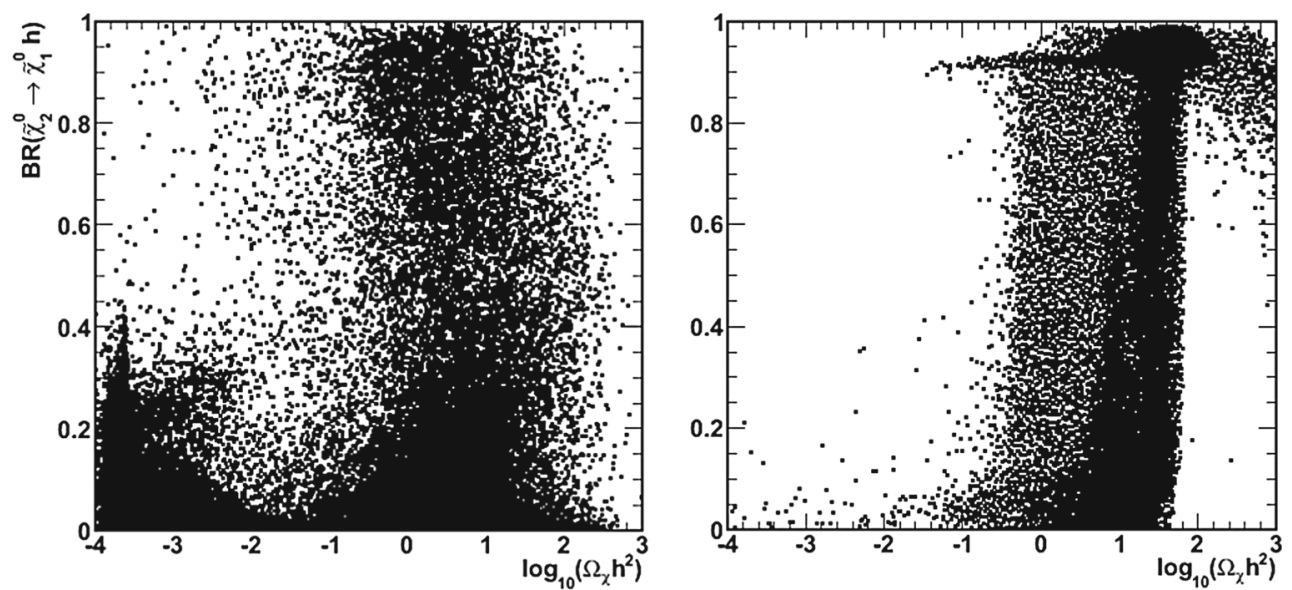

Fig. 5 Branching fraction for $\tilde{\chi}_{2}^{0} \rightarrow h \tilde{\chi}_{1}^{0}$ as a function of the neutralino relic density, $\Omega_{\chi} h^{2}$, for pMSSM (left) and CMSSM (right) points

hilate through the $A^{0}$ pole to acquire a relic density in agreement with the CMB data $[64,65]$. It is therefore important to reconsider the apparent dominance of the $\tilde{\chi}_{2}^{0} \rightarrow h \tilde{\chi}_{1}^{0}$ decay in the CMSSM, when examining the SUSY phenomenology in the context of more general models, or even in the CMSSM itself after imposing the dark matter relic density constraint. Still, the process of $h$ production in neutralino decays is of crucial importance in specific regions of the parameter space, which are largely complementary to those yielding decays into sleptons or $Z$ bosons, probed by the multi-lepton analyses at the LHC.

\section{Neutralino decays to $h$ : experimental searches}

In the following we discuss the perspectives for investigating these decays at the LHC and at an $e^{+} e^{-}$linear collider (LC). We consider for the LHC an integrated luminosity of $25 \mathrm{fb}^{-1}$ at $8 \mathrm{TeV}, 300 \mathrm{fb}^{-1}$ and $3000 \mathrm{fb}^{-1}$ at $14 \mathrm{TeV}$. For the LC we consider $500 \mathrm{fb}^{-1}$ of integrated luminosity at $\sqrt{s}=0.5 \mathrm{TeV}$ and $1 \mathrm{ab}^{-1}$ at $1,1.5$ and $3 \mathrm{TeV}$. For each of these scenarios, we combine the decay branching fractions with the relevant neutralino production cross sections to study the number of expected events and compare them to the expected backgrounds. For the LHC, we compare the regions of exclusion from the $b b \ell$ mode to the two- and three-lepton channels.

\subsection{LHC}

As a first step, we combine the results on the branching fractions discussed in the previous section to the expected relevant cross sections for the associated chargino-neutralino production. Through the decay $\tilde{\chi}_{1}^{ \pm} \rightarrow W^{ \pm} \tilde{\chi}_{1}^{0}, W^{ \pm} \rightarrow \ell^{ \pm} v$, this process produces a high $p_{T}$ lepton, which is very valuable for the event trigger and subsequent selection. The cross sections for chargino-neutralino production at 8 and $14 \mathrm{TeV}$, followed by $\tilde{\chi}_{2,3}^{0} \rightarrow h \tilde{\chi}_{1}^{0} ; h \rightarrow b \bar{b}, \tau \tau$ and $\tilde{\chi}_{1}^{ \pm} \rightarrow W^{ \pm} \tilde{\chi}_{1}^{0}$, $W^{ \pm} \rightarrow \ell^{ \pm} v$ are given in Figs. 6 and 7 , respectively. Because of the small values of these cross sections and the large spread for a given neutralino mass, luminosity is as important as energy for probing electroweak SUSY particle production at the LHC. The high-luminosity LHC program (HLLHC) bringing a tenfold increase in the data statistics will be extremely beneficial to these studies.

The ATLAS and CMS experiments have conducted searches for electroweak production of SUSY particles in the two- and three-lepton + MET channels and the $b b \ell+$ MET channel. Accepted pMSSM scan points fulfilling the selection criteria discussed above and having an inclusive chargino and neutralino production cross section yielding at least ten signal events at 8 or $14 \mathrm{TeV}$ collision energies are studied in detail. A set of 20k SUSY events with inclusive neutralino and chargino production is generated for each of these points at both centre-of-mass energies. Simulated events are passed through a fast parametric simulation using the Delphes 3.0 package which simulates the physics objects used in the subsequent analysis. Jets are reconstructed using the anti-kt algorithm [66], implemented in the FastJet package [67]. The selection criteria of the two- and three-lepton + MET $[17,20]$ and the $b b \ell+$ MET [21] analyses of ATLAS are applied. The exclusion of the pMSSM points is assessed using the CLs technique [68], where the number of expected signal events is obtained from the result of the selection on the generated events and that of background events is taken from that estimated for the ATLAS analyses, appropriately rescaled to the assumed luminosity and collision energy. The results of this simulation are validated by comparing the $95 \%$ C.L. exclusion contours with those expected for the ATLAS analyses, under the same assumptions. These contours agree within 20-30\%.

We study the fraction of accepted pMSSM points for which the different channels and their combination lead to 

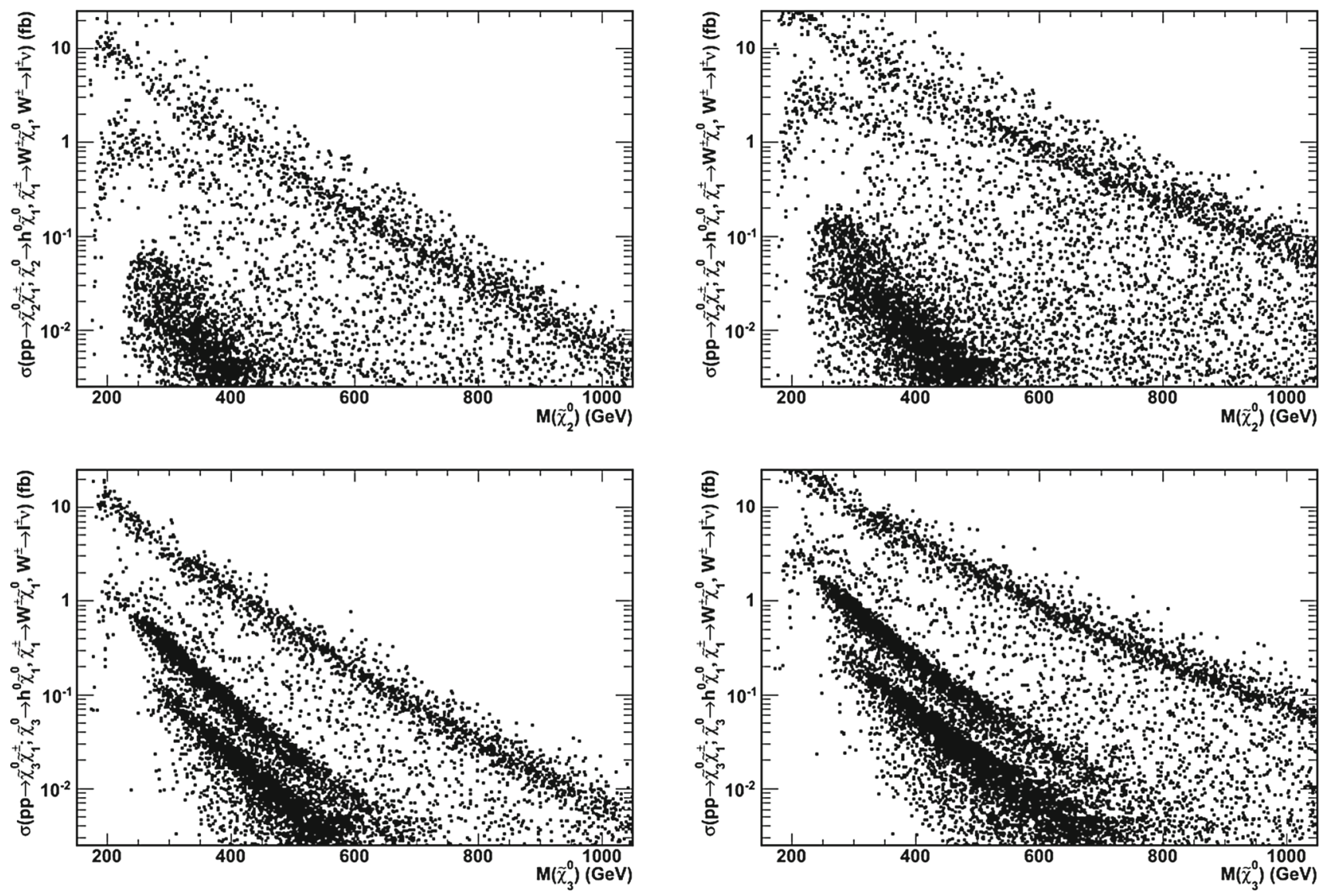

Fig. 6 Cross section for $\tilde{\chi}_{1}^{ \pm} \tilde{\chi}_{2}^{0}$ (upper) and $\tilde{\chi}_{1}^{ \pm} \tilde{\chi}_{3}^{0}$ (lower) production vs. $M_{\chi_{2}^{0}}$ and $M_{\chi_{3}^{0}}$, respectively, with $\tilde{\chi}_{2,3}^{0} \rightarrow h \tilde{\chi}_{1}^{0} ; h \rightarrow b b, \tau \tau$ and $\tilde{\chi}_{1}^{ \pm} \rightarrow W^{ \pm} \tilde{\chi}_{1}^{0} ; W^{ \pm} \rightarrow \ell^{ \pm} v$ in $8 \mathrm{TeV} p p$ collisions for a set of accepted pMSSM points

a $95 \%$ C.L. exclusion for $25 \mathrm{fb}^{-1}$ at $8 \mathrm{TeV}, 300 \mathrm{fb}^{-1}$ and $3000 \mathrm{fb}^{-1}$ at $14 \mathrm{TeV}$. The fractions of the accepted pMSSM points excluded at 8 and $14 \mathrm{TeV}$ by the $b b \ell+$ MET channel in the $\left[|\mu|,\left|M_{1}\right|\right]$ plane are given in Fig. 8. These fractions for the different channels over the full scan range are compared in Table 1. Because the product of the $\tilde{\chi}_{1}^{ \pm} \tilde{\chi}_{2,3}^{0}$ production cross section and decay branching fraction into $h$ is of $\mathcal{O}(0.1 \mathrm{fb})$, or less, at $8 \mathrm{TeV}$, the Run-1 LHC searches have just scraped the region of interest for these processes in the MSSM. The Run-2 searches will definitely attain an interesting sensitivity for neutralino masses below $600-700 \mathrm{GeV}$, as can be seen in Fig. 8. The values of the fractions of pMSSM points depend on the ranges for the pMSSM parameters adopted in the scan, given at Eq. (1). However, the location of the regions of larger sensitivity, highlighted by the large fractions of excluded pMSSM points, are of general validity. The MSSM parameter coverage provided by SUSY weak production searches at $8 \mathrm{TeV}$ appears, in general, to be marginal. Of our accepted scan points with SUSY masses up to $3 \mathrm{TeV}$, only $0.3 \%$ are excluded by the combinations of the three channels

Fig. 7 Same as Fig. 6 for $14 \mathrm{TeV} p p$ collisions

considered here. These are also independently excluded by searches in jets + MET and monojet channels. The factor of $\sim 3$ increase of the $p p \rightarrow \tilde{\chi}_{1}^{ \pm} \tilde{\chi}_{2,3}^{0}$ production cross section and the factor of $\sim 10-100$ increase in the assumed statistics, make the coverage of the parameter space to expand significantly when moving from $25 \mathrm{fb}^{-1}$ at $8 \mathrm{TeV}$ to $300 \mathrm{fb}^{-1}$ and $3000 \mathrm{fb}^{-1}$ at $14 \mathrm{TeV}$, with $3 \%$ of the pMSSM points expected to be excluded with the HL-LHC data.

The leptonic channels are sensitive to the contribution of light sleptons in the decays $\tilde{\chi}_{2}^{0} \rightarrow \tilde{\ell} \ell ; \tilde{\ell} \rightarrow \ell \tilde{\chi}_{1}^{0}$, and of $Z$ bosons in the decay $\tilde{\chi}_{2}^{0} \rightarrow Z \tilde{\chi}_{1}^{0} ; Z \rightarrow \ell \ell$. In the unconstrained MSSM, slepton masses can be pushed well above the $\tilde{\chi}_{2}^{0}$ mass, so that the only remaining dominant two-body $\tilde{\chi}_{2}^{0}$ decays are either $h \tilde{\chi}_{1}^{0}$ or $Z \tilde{\chi}_{1}^{0}$. This motivates the pursue of the study of the challenging $h \tilde{\chi}_{1}^{0}$ channel, through the $b \bar{b} \ell+E_{T}^{\text {missing }}$ topology, which complements the $Z \tilde{\chi}_{1}^{0}$ channel and provides an increasing fraction of the LHC sensitivity to chargino-neutralino pair production as the energy and integrated luminosity increase. The neutralino decay into $h$ accounts for approximately one third of the excluded points with $25 \mathrm{fb}^{-1}$ at $8 \mathrm{TeV}$ to more than half of them for $3000 \mathrm{fb}^{-1}$ at $14 \mathrm{TeV}$. The $h$ decay into $\tau \tau$ pairs also leads to the same $\tau^{+} \tau^{-} \ell+E_{T}^{\text {missing }}$ final state as $Z \tilde{\chi}_{1}^{0} ; Z \rightarrow \tau \tau$. If a signal 
is observed in the $b \bar{b} \ell+E_{T}^{\text {missing }}$ and/or $\tau^{+} \tau^{-} \ell+E_{T}^{\text {missing }}$ modes it would become essential to attempt to identify the contributing channels, possibly through a fit to the $b b$ invariant mass, as discussed below, and the $\tau \tau$ transverse mass.

The nature of the lightest neutralino has been analysed for the points excluded at 8 and $14 \mathrm{TeV}$ and the results are summarised in Table 2. As expected, the largest fraction of points has bino $\tilde{\chi}_{1}^{0}$, since this is dominant for light $\tilde{\chi}_{1}^{0}$ states, followed by higgsino-gaugino mixed states, which become increasingly important as the search sensitivity expands with higher energy and larger data sets.

If a signal is observed in the $b b \ell+$ MET channel in the $13-14 \mathrm{TeV}$ LHC runs, some important measurements could be performed. In the case the $\tilde{\chi}_{2}^{0}$ and $\tilde{\chi}_{3}^{0}$ states are close in mass, as expected over a large part of the MSSM parameter space, they would be both produced at the LHC and may subsequently decay into $h \tilde{\chi}_{1}^{0}$ and $Z \tilde{\chi}_{1}^{0}$, as discussed above. In this case, the $b \bar{b} \ell+E_{T}^{\text {missing }}$ final state will receive contributions from both $h \rightarrow b \bar{b}$ and $Z \rightarrow b \bar{b}$, which can be resolved, at least on a statistical basis, from an analysis of the invariant mass of the reconstructed $b b$ system. The sensitivity of this analysis is assessed using an inclusive sample of $p p \rightarrow \tilde{\chi}_{1}^{ \pm} \tilde{\chi}_{2,3}^{0}$ generated at $14 \mathrm{TeV}$ with an equivalent luminosity of $300 \mathrm{fb}^{-1}$ for a pMSSM point with the $\tilde{\chi}_{1}^{ \pm}, \tilde{\chi}_{2}^{0}$ and $\tilde{\chi}_{3}^{0}$ at masses between 420 and $450 \mathrm{GeV}$. Events are reconstructed in the $b \bar{b} \ell+E_{T}^{\text {missing }}$ and those with one isolated lepton, two $b$-tagged jets and $E_{T}^{\text {missing }}>50 \mathrm{GeV}$ are considered. The jet $b$-tagging efficiency is assumed to be 0.75 . The invariant mass distribution of the $b$ jets is shown in Fig. 9. It receives contributions from the two dominant decays of the MSSM point chosen for simulation, $\chi_{2}^{0} \rightarrow Z \tilde{\chi}_{1}^{0}, Z \rightarrow b \bar{b}$ and $\chi_{3}^{0} \rightarrow h \tilde{\chi}_{1}^{0}, h \rightarrow b \bar{b}$ and the two sub-leading decays $\chi_{3}^{0} \rightarrow Z \tilde{\chi}_{1}^{0}, Z \rightarrow b \bar{b}$ and $\chi_{2}^{0} \rightarrow h \tilde{\chi}_{1}^{0}, h \rightarrow b \bar{b}$. From the branching fractions and cross sections of this specific pMSSM point, the fraction of $h$ in these decays is 0.73 .

The experimental di-jet mass resolution $\delta M / M$ for the $b b$ system at the $Z$ and $h$ masses is $\sim 0.13$ from the Delphes simulation, which agrees with the performance obtained on full simulation for the LHC $H_{S M} \rightarrow b \bar{b}$ searches. A $\chi^{2}$ fit is performed on the reconstructed invariant mass distribution to extract the fraction of decays into $h \tilde{\chi}$. The contribution from $Z$ and $h$ is modelled by Breit-Wigner distributions folded with a Gaussian resolution term and the combinatorial background is described by a first order polynomial. The $Z$ and $h$ fractions and the background parameters are left free in the fit. The result gives a fraction of decays with an $h$ boson of $0.65 \pm 0.09$, which agrees with the generated values within the statistical uncertainty. With the tenfold increase in data statistics expected by the HL-LHC program, the relative statistical uncertainty should be reduced from 14 to $\sim 4 \%$, if the event reconstruction and $b$-tagging capabilities are maintained in the higher pile-up environment of the HL-LHC.
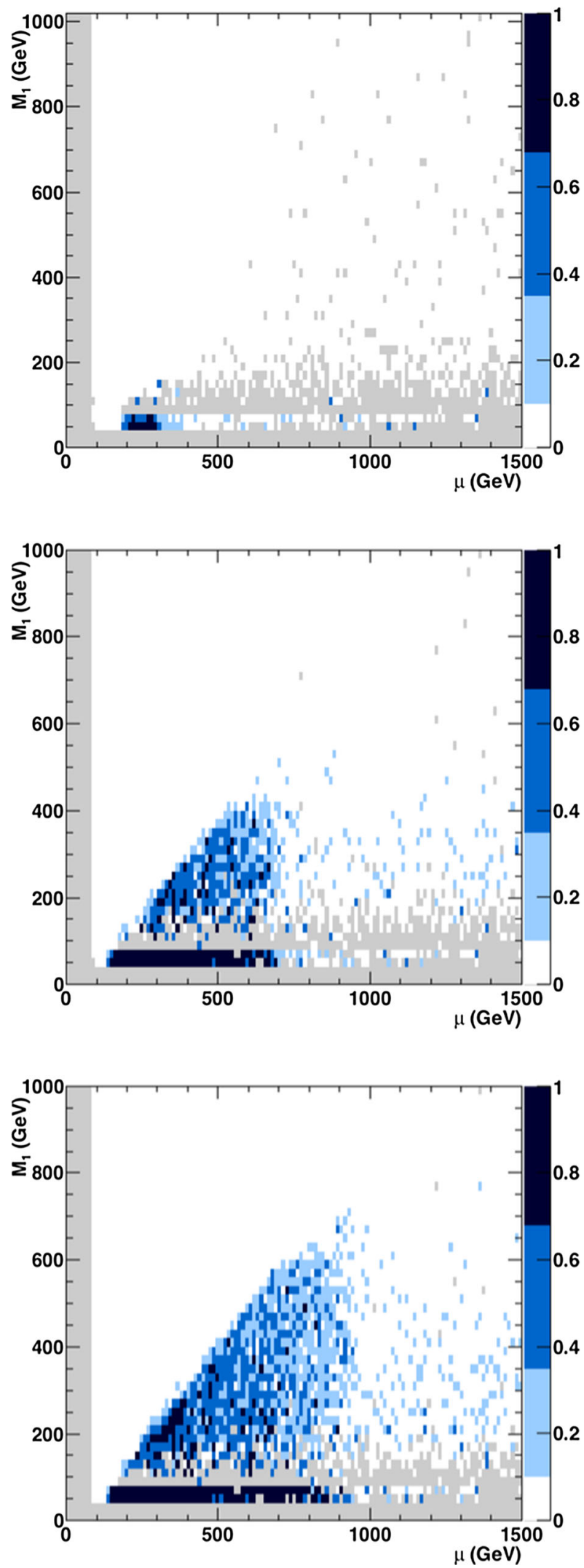

Fig. 8 Fraction of accepted pMSSM points in the $\left[|\mu|,\left|M_{1}\right|\right]$ plane excluded by the $b b \ell+$ MET channel at $8 \mathrm{TeV}$ (upper) and their extrapolations at $14 \mathrm{TeV}$ for $300 \mathrm{fb}^{-1}$ (centre) and $3000 \mathrm{fb}^{-1}$ (lower) 
Table 1 Fraction of pMSSM points excluded in the various channels at 8 and $14 \mathrm{TeV}$

\begin{tabular}{llll}
\hline & $\begin{array}{l}8 \mathrm{TeV} \\
25 \mathrm{fb}^{-1}\end{array}$ & $\begin{array}{l}14 \mathrm{TeV} \\
300 \mathrm{fb}^{-1}\end{array}$ & $\begin{array}{l}14 \mathrm{TeV} \\
3000 \mathrm{fb}^{-1}\end{array}$ \\
\hline $2 / 3 \ell$ & 0.002 & 0.008 & 0.016 \\
$3 \ell(\mathrm{Z})$ & 0.001 & 0.004 & 0.009 \\
$b b(\mathrm{~h}) \ell$ & 0.001 & 0.009 & 0.015 \\
All EWK & 0.003 & 0.015 & 0.029 \\
\hline
\end{tabular}

Table 2 Neutralino LSP nature for the pMSSM points excluded at 8 and $14 \mathrm{TeV}$

\begin{tabular}{llll}
\hline & $\begin{array}{l}8 \mathrm{TeV} \\
25 \mathrm{fb}^{-1}\end{array}$ & $\begin{array}{l}14 \mathrm{TeV} \\
300 \mathrm{fb}^{-1}\end{array}$ & $\begin{array}{l}14 \mathrm{TeV} \\
3000 \mathrm{fb}^{-1}\end{array}$ \\
\hline Bino LSP & 0.789 & 0.706 & 0.679 \\
Wino LSP & 0.0 & 0.0 & 0.010 \\
Higgsino LSP & 0.0 & 0.0 & 0.0 \\
Mixed LSP & 0.211 & 0.294 & 0.311 \\
\hline
\end{tabular}

\section{$6.2 e^{+} e^{-}$linear collider}

An $e^{+} e^{-}$collider of sufficient energy and luminosity is very well suited for reconstructing two-body decays of neutralinos, pair produced in the process $e^{+} e^{-} \rightarrow \tilde{\chi}_{i}^{0} \tilde{\chi}_{j}^{0}$, including those decaying into $h$ and $Z$ bosons. In particular, since the energy of the produced $\tilde{\chi}_{i}^{0}$ is known, apart from beam radiation effects, the energy distribution of the reconstructed bosons can be used to precisely determine the $\tilde{\chi}_{i}^{0}$ mass. However, pair production cross sections are typically small. The production of neutralino pairs in $e^{+} e^{-}$collisions is mediated by $s$-channel $Z$ boson exchanges as well as $t$ - and $u$-channel left- and right-handed $\tilde{e}$ exchange. If the sleptons are assumed to be very heavy or the produced neutralinos are higgsinolike (and thus the coupling $\tilde{\chi}^{0} \tilde{e} e$ is negligibly small) only the $s$-channel $Z$ boson exchange is relevant. In fact, the neutralino cross sections are rather small, even in the case of $\tilde{\chi}_{1}^{0} \tilde{\chi}_{2}^{0}$ production where the phase space is more favourable. The only exception is when both $\tilde{\chi}_{1}^{0}$ and $\tilde{\chi}_{2}^{0}$ are higgsino-like and have maximal couplings to the $Z$ boson. Also the cross sections with identical neutralinos are extremely small. Due to Fermi statistics, the neutralinos are produced in $p$-waves and therefore suppressed at threshold and, in the case of gauginos or higgsinos, the cross section is further suppressed by the couplings $\mathcal{Z}_{i i} \propto N_{i 3}^{2}-N_{i 4}^{2}$. The cross sections for mixed production of $\tilde{\chi}_{1}^{0} \tilde{\chi}_{3}^{0}, \tilde{\chi}_{2}^{0} \tilde{\chi}_{3}^{0}$ and $\tilde{\chi}_{3}^{0} \tilde{\chi}_{4}^{0}$ are significant, except in the case where one of the neutralinos is a pure gaugino, which leads to suppressed $Z \tilde{\chi} \tilde{\chi}$ couplings.

The product of the pair production cross sections and the $\tilde{\chi}_{2,3}^{0} \rightarrow h \tilde{\chi}_{1}^{0}$ branching fractions is shown in Fig. 10 as a function of the neutralino mass, for the four $\sqrt{s}$ energy values $(0.5,1,1.5$ and $3 \mathrm{TeV})$ chosen for this study.

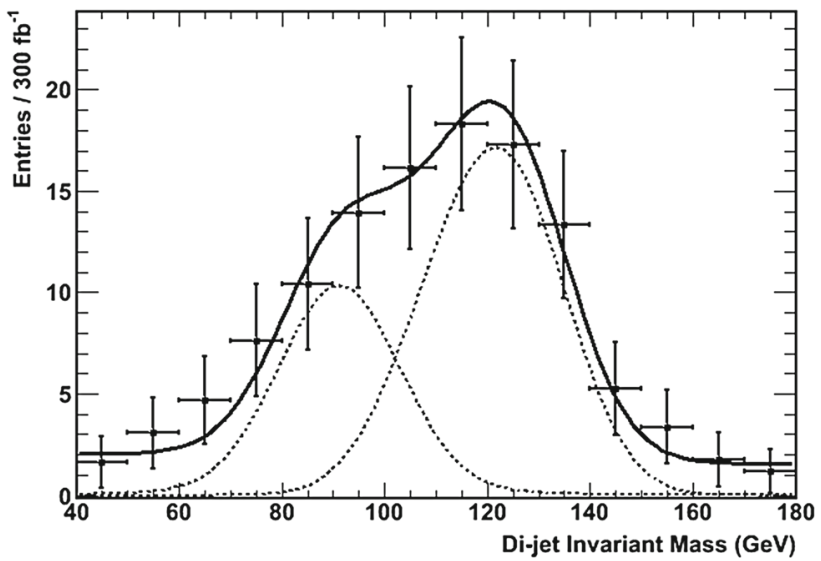

Fig. 9 Di-jet invariant mass for $2 b$ jets + lepton + missing energy $p p \rightarrow \chi_{1}^{ \pm} \chi_{2,3}^{0}$ events produced at $14 \mathrm{TeV}$. The result of the fit is shown by the continuous line, with the $Z$ and $h$ components represented by the dotted lines
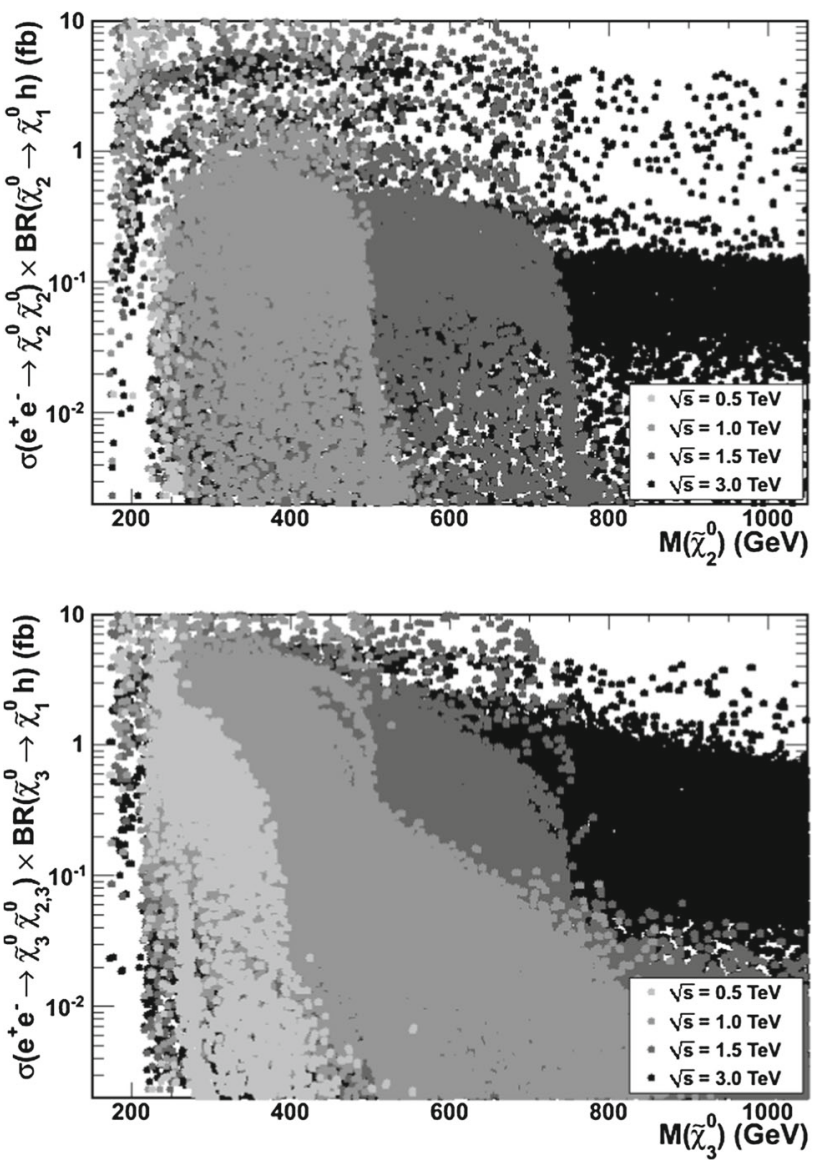

Fig. 10 Product of neutralino pair production cross section and neutralino decay branching fraction into $h \tilde{\chi}_{1}^{0}$ as a function of the $\tilde{\chi}_{2}^{0}$ (upper) and $\tilde{\chi}_{3}^{0}$ (lower) masses at $\sqrt{s}=0.5,1,1.5$ and $3 \mathrm{TeV}$ shown by the dots in graded tones of grey

It is important to observe that, in the region of parameters where the $\tilde{\chi}_{2}^{0} \rightarrow h \tilde{\chi}_{1}^{0}$ process is the dominant neutralino decay, the $\tilde{\chi}_{2}^{0}$ tends to decouple from the $Z$. This sup- 


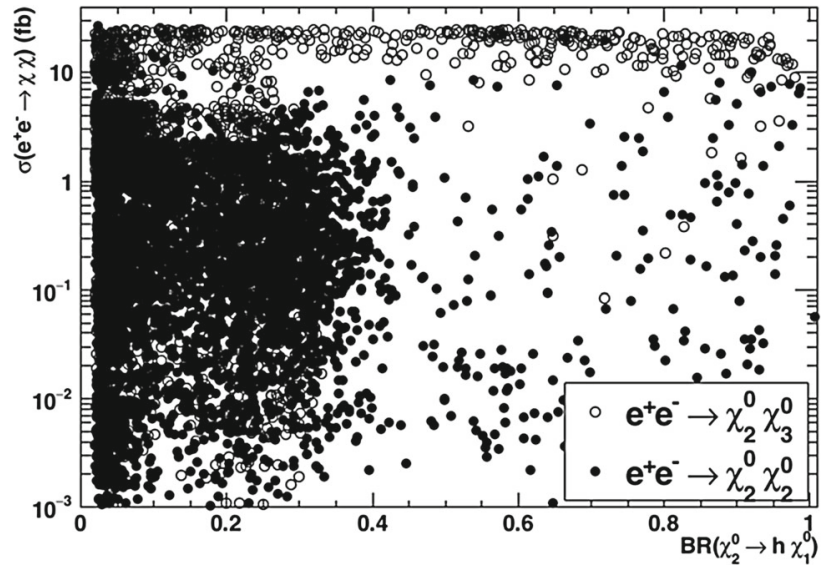

Fig. 11 Neutralino pair production cross section for $e^{+} e^{-} \rightarrow \tilde{\chi}_{2}^{0} \tilde{\chi}_{2}^{0}$ (filled circles) and $\tilde{\chi}_{2}^{0} \tilde{\chi}_{3}^{0}$ (open circles) at $\sqrt{s}=1.5 \mathrm{TeV}$ shown as a function of the $\tilde{\chi}_{2}^{0} \rightarrow h \tilde{\chi}_{1}^{0}$ branching fraction for pMSSM points where the sum of the neutralino masses, $2 M_{\tilde{\chi}_{2}^{0}}$ and $M_{\tilde{\chi}_{2}^{0}}+M_{\tilde{\chi}_{3}^{0}}$, respectively, does not exceed $1.4 \mathrm{TeV}$

presses the $e^{+} e^{-} \rightarrow \tilde{\chi}_{2}^{0} \tilde{\chi}_{2}^{0}$ pair production, leaving instead the $e^{+} e^{-} \rightarrow \tilde{\chi}_{2}^{0} \tilde{\chi}_{3}^{0}$ process as the only significant neutralino production mechanism, when kinematically allowed. This correlation between pair production cross section and decay branching fractions is illustrated in Fig. 11 for a sample of accepted pMSSM points, where the sum of the masses of the pair-produced neutralinos is more than $100 \mathrm{GeV}$ below the collision energy, to avoid threshold effects. It is evident that the region of large branching fractions into $h$ bosons is dominated by larger $\tilde{\chi}_{2}^{0} \tilde{\chi}_{3}^{0}$ production.

Chargino and neutralino pair production can be typically observed at a linear collider operating at a centre-of-mass energy of $\sqrt{s}$ for $\mu$ and $M_{2}$ values up to the kinematic limit of $\sqrt{s} / 2$. This makes the sensitivity of a LC, already at $0.5 \mathrm{TeV}$ and more definitely at $1.0 \mathrm{TeV}$ and above, highly complementary to that of the LHC at $14 \mathrm{TeV}$. Due to the relatively large mass splitting from the LSP required for sensitivity in the $\tilde{\chi}_{1}^{ \pm} \tilde{\chi}_{2}^{0}$ production process, the parameter region where the LHC may detect chargino-neutralino production starts at $M_{2}>250 \mathrm{GeV}$ and $\mu>250 \mathrm{GeV}$. The LC operating already at $0.5 \mathrm{TeV}$ will completely cover the complementary region of $M_{2}<250 \mathrm{GeV}$ or $\mu<250 \mathrm{GeV}$. The decay $\tilde{\chi}_{2}^{0} \rightarrow Z \tilde{\chi}_{1}^{0}$ was studied as a benchmark reaction at the ILC at $\sqrt{s}=0.5 \mathrm{TeV}$ using full simulation and reconstruction as part of the ILC LoIs $[69,70]$. In these analyses, the $\tilde{\chi}_{2}^{0}$ mass of $217 \mathrm{GeV}$ was determined with a statistical relative accuracy of $1 \%$. The decays $\tilde{\chi}_{2}^{0} \rightarrow h \tilde{\chi}_{1}^{0}$ and $\tilde{\chi}_{2}^{0} \rightarrow Z \tilde{\chi}_{1}^{0}$ have been studied for CLIC at $\sqrt{s}=3 \mathrm{TeV}$ [71]. The $\tilde{\chi}_{2}^{0} \rightarrow h \tilde{\chi}_{1}^{0}$ decay, followed by $h \rightarrow b \bar{b}$, leads to the signature $b \bar{b} b \bar{b}+$ missing energy final state. The $\tilde{\chi}_{2}^{0}$ decay can be isolated in an inclusive SUSY sample, where its mass and the $h$ yield are determined with a relative statistical accuracy of 4 and $5 \%$, respectively.
With an expected integrated luminosity of $0.5-3 \mathrm{ab}^{-1} \mathrm{a}$ high energy linear collider can observe these decays over a significant part of the kinematically allowed region of the parameter space. This is quantified here by the fraction of the pMSSM points yielding at least 25 signal events for an integrated luminosity of $0.5 \mathrm{ab}^{-1}$ for $\sqrt{s}=0.5 \mathrm{TeV}$ and of $1 \mathrm{ab}^{-1}$ for $1 \mathrm{TeV}, 2 \mathrm{ab}^{-1}$ for $1.5 \mathrm{TeV}$ and $3 \mathrm{ab}^{-1}$ for $3 \mathrm{TeV}$, over the $\left[\mu, M_{1}\right]$ parameter space, as shown in Fig. 12, where the sensitivity to the decay into $h$ is compared to that of inclusive neutralino and chargino decays.

The background for these decays with two $h$ or a $h$ and a $Z$ bosons and large missing energy are expected to be small and this justifies our simple criteria of event counting to obtain the regions of sensitivity in the $\left[\mu, M_{1}\right]$ plane. These backgrounds have been studied in more detail at $3 \mathrm{TeV}$, as discussed below.

Once a signal is observed, the yield of $h$ bosons can be determined in an inclusive SUSY sample. We study it for the specific case of a MSSM model having $\tilde{\chi}_{1}^{0}$ with mass of $340 \mathrm{GeV}, \tilde{\chi}_{2}^{0}$ with mass of $643 \mathrm{GeV}$ decaying predominantly into $h \tilde{\chi}_{1}^{0}$ and $\tilde{\chi}_{1}^{ \pm}$with mass of $643 \mathrm{GeV}$ decaying exclusively into $W^{ \pm} \tilde{\chi}_{1}^{0}$ and $M_{h}=126.1 \mathrm{GeV}$, in $3 \mathrm{TeV} e^{+} e^{-}$ collisions. An inclusive sample of SUSY events, corresponding to $0.5 \mathrm{fb}^{-1}$ of integrated luminosity, is generated using Pythia and events are fully simulated using Geant - 4 [72] and reconstructed following the same analysis procedure discussed in [71]. Events are pre-selected requiring a visible energy $0.08 \sqrt{s}<E_{\text {tot }}<0.6 \sqrt{s}$, an energy in charged particles larger than $150 \mathrm{GeV}$, transverse energy larger than $200 \mathrm{GeV}$ and jet multiplicity $2 \leq N_{\text {jets }}<5$. Jet clustering is performed using the Durham jet algorithm [73], with $y_{c u t}=$ 0.0025 , on the reconstructed particle flow objects of the Pandora particle flow package [74]. Selected events are clustered into four jets and the di-jet invariant mass for all the three possible pairings is computed. The jet pairing minimising the difference between the di-jet invariant masses is selected, provided the mass difference is below $20 \mathrm{GeV}$. The fraction of background non-resonant events is obtained from the di-jet mass side-bands $20<E_{j j}<60 \mathrm{GeV}$ and $140<E_{j j}<200 \mathrm{GeV}$. The fraction of $W^{+} W^{-}, Z Z$ and $h h$ events in the inclusive 4-jet SUSY events is extracted by a $\chi^{2}$ fit to this di-jet mass distribution. The $W^{ \pm}$and $Z$ mass peaks are parametrised as Breit-Wigner functions convoluted with a Gaussian term describing the experimental resolution. The mass and width values of the Breit-Wigner functions are fixed to their generated values, while the total area and the width of the Gaussian resolution terms are left free in the fit.

The $h$ peak is modelled as the sum of two Gaussian curves, one representing the correctly reconstructed signal events, centred at the nominal $M_{h}$ value, the second describing decays where the mass has a lower reconstructed value due to semi-leptonic $b$ decays. The central value, width and fraction of events in this second Gaussian is extracted by a 

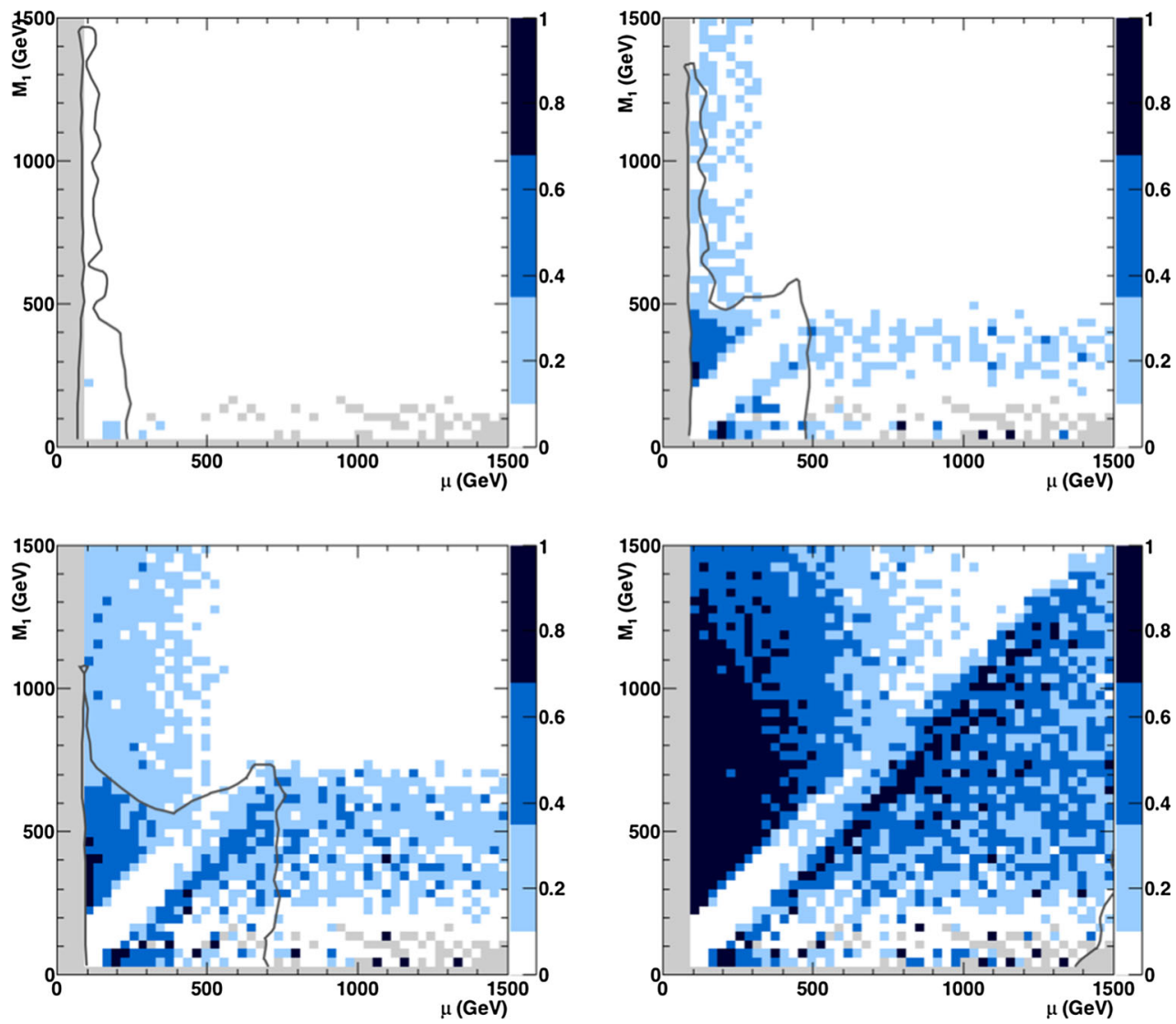

Fig. 12 Fraction of accepted pMSSM points in the $\left[\mu, M_{1}\right]$ plane giving at least 25 events in the chargino and neutralino pair production channels with $\tilde{\chi}_{2,3}^{0} \rightarrow h \tilde{\chi}_{1}^{0}$ for $0.5 \mathrm{ab}^{-1}$ of $e^{+} e^{-}$data at $\sqrt{s}=0.5 \mathrm{TeV}$ (upper-left), $1 \mathrm{ab}^{-1}$ at $\sqrt{s}=1 \mathrm{TeV}$ (upper-right), $2.0 \mathrm{ab}^{-1}$ at $\sqrt{s}=$

$1.5 \mathrm{TeV}$ (lower left) and $3 \mathrm{ab}^{-1}$ at $\sqrt{s}=3 \mathrm{TeV}$ (lower-right). The grey lines indicate the regions where more than $68 \%$ of the accepted pMSSM points give at least 25 signal events in all chargino and neutralino pair production channels

fit to a pure sample of decays into bosons and fixed in the fit, while the Gaussian width of the main peak is kept free. The yield of $h$ bosons, with mass $126.1 \mathrm{GeV}$, is extracted by a $\chi^{2}$ fit to the di-jet mass distribution of events in 4-jet final states, shown in Fig. 13 for all di-jets and for those passing di-jet b-tagging based on the ZVTOP algorithm [75] implemented in the LCFIVertex package [76]. We measure the fraction of $h$ bosons to be $0.269 \pm 0.013$ and that of $Z$ bosons to be $0.037 \pm 0.016$, after the non-resonant background subtraction, which compares well to the original values of 0.290 and 0.025 , respectively, of the generated events. This specific example shows the feasibility to accurately determine the yield of $h$ bosons in SUSY particle decays in the data of an $e^{+} e^{-}$collider operating at sufficient energy.

\section{Conclusions}

The observation of a Higgs particle with a mass of $\simeq 126 \mathrm{GeV}$ promotes the exploration of its possible role in the decay of

weakly interacting particles in the context of supersymmetry. Neutralino decays offer a prime opportunity, since the $\tilde{\chi}_{2,3}^{0} \rightarrow h \tilde{\chi}_{1}^{0}$ process provides the largest yield of $h$ bosons in SUSY decays in the MSSM and this decay channel is highly complementary to channels leading to multi-lepton final states, such as $Z$ and $\tilde{\ell} \ell$. The typical values of cross sections times decay branching fractions to $b b \ell$ final state are of the order $0.01-1 \mathrm{fb}$ in $8 \mathrm{TeV} p p$ collisions and a factor of about three larger at $14 \mathrm{TeV}$ for $M_{\chi_{2,3}^{0}} \leq 600 \mathrm{GeV}$. The LHC experiments have just started probing these processes. Extrapolating to $13-14 \mathrm{TeV}$ the preliminary results obtained in the first searches with the $b b \ell+$ MET channel at $8 \mathrm{TeV}$ shows that the increase in cross section and available statistics should make possible to probe a significant region of the MSSM parameter space. At 13-14 TeV, the neutralino decays into Higgs should account for more than half of the pMSSM points for which the electroweak $\tilde{\chi}_{1}^{ \pm} \tilde{\chi}_{2,3}^{0}$ production channels have sensitivity. The points accessible through the $h$ channel belong to regions complementary to those covered by searches with multi-leptons. If a signal is 

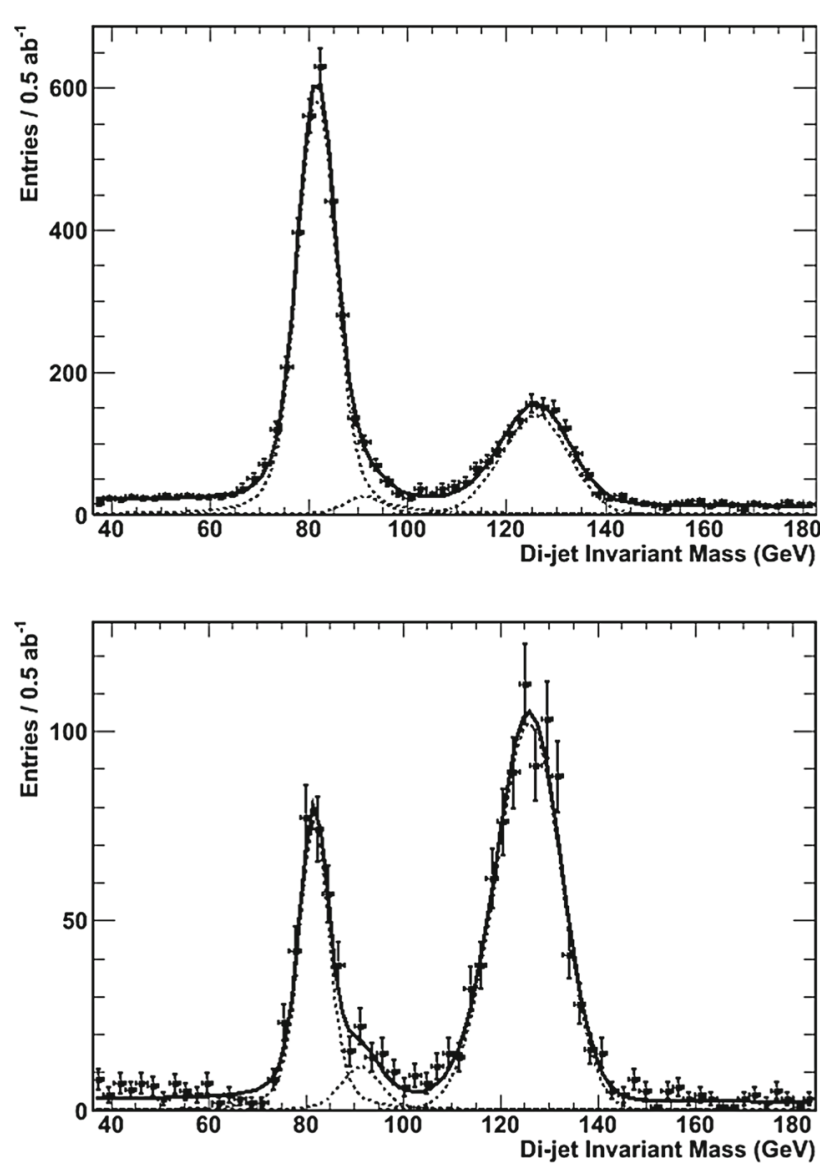

Fig. 13 Di-jet invariant mass in inclusive 4 -jet + missing energy SUSY events produced in $\sqrt{s}=3 \mathrm{TeV} e^{+} e^{-}$collisions for all selected events (upper) and $b$-tagged events (lower). The result of the fit is shown by the continuous line with the individual $W, Z$ and $h$ components represented by the dotted lines

observed, then the fraction of $h$ bosons produced in neutralino decays could be measured to a statistical accuracy of better than $10 \%$ at the HL-LHC. An $e^{+} e^{-}$linear collider of sufficient energy, $\sqrt{s} \geq 1 \mathrm{TeV}$, and luminosity, yielding $\geq 1.0-3.0 \mathrm{ab}^{-1}$ of data, can study chargino-neutralino pair production in regions of the parameter space highly complementary to the LHC at $14 \mathrm{TeV}$. Further, it can study $h$ production in neutralino decays for masses up to the kinematical limit for pair production, obtaining $\mathcal{O}(1 \%)$ precision on the mass and $\mathcal{O}(5 \%)$ on the yield of Higgs bosons from neutralino decays in inclusive multi-jet SUSY events.

Acknowledgments We thank A. Djouadi for extensive discussion and A. Canepa and Z. Gecse for useful suggestions. M.B. wishes to thank the Galileo Galilei Institute for Theoretical Physics for the hospitality and INFN for partial support during the development of this study.

Open Access This article is distributed under the terms of the Creative Commons Attribution License which permits any use, distribution, and reproduction in any medium, provided the original author(s) and the source are credited.

Funded by $\mathrm{SCOAP}^{3}$ / License Version CC BY 4.0.

\section{References}

1. G. Aad et al. (ATLAS Collaboration), Phys. Lett. B 716, 1 (2012). arXiv:1207.7214 [hep-ex]

2. S. Chatrchyan et al., CMS Collaboration, Phys. Lett. B 716, 30 (2012). arXiv:1207.7235 [hep-ex]

3. J.F. Gunion, H.E. Haber, R.M. Barnett, M. Drees, D. Karatas, X. Tata, H. Baer, Int. J. Mod. Phys. A 2, 1145 (1987)

4. J.F. Gunion, H.E. Haber, Phys. Rev. D 37, 2515 (1988)

5. A. Bartl, W. Majerotto, N. Oshimo, Phys. Lett. B 216, 233 (1989)

6. A. Djouadi, Y. Mambrini, M. Muhlleitner, Eur. Phys. J. C 20, 563 (2001). hep-ph/0104115

7. A. Datta, A. Djouadi, M. Guchait, F. Moortgat, Nucl. Phys. B 681, 31 (2004). hep-ph/0303095

8. K. Huitu, R. Kinnunen, J. Laamanen, S. Lehti, S. Roy, T. Salminen, Eur. Phys. J. C 58, 591 (2008). arXiv:0808.3094 [hep-ph]

9. S. Gori, P. Schwaller, C.E.M. Wagner, Phys. Rev. D 83, 115022 (2011). arXiv:1103.4138 [hep-ph]

10. O. Stal, G. Weiglein, JHEP 1201, 071 (2012). arXiv:1108.0595 [hep-ph]

11. K. Howe, P. Saraswat, JHEP 1210, 065 (2012). arXiv:1208.1542 [hep-ph]

12. G.D. Kribs, A. Martin, T.S. Roy, M. Spannowsky, Phys. Rev. D 81, 111501 (2010). arXiv:0912.4731 [hep-ph]

13. G.D. Kribs, A. Martin, T.S. Roy, M. Spannowsky, Phys. Rev. D 82, 095012 (2010). arXiv:1006.1656 [hep-ph]

14. D. Ghosh, M. Guchait, D. Sengupta, Eur. Phys. J. C 72, 2141 (2012). arXiv:1202.4937 [hep-ph]

15. G. Aad et al., ATLAS Collaboration, Phys. Lett. B 718, 879 (2013). arXiv: 1208.2884 [hep-ex]

16. S. Chatrchyan et al., CMS Collaboration, JHEP 1211, 147 (2012). arXiv:1209.6620 [hep-ex]

17. G. Aad et al., ATLAS Collaboration, JHEP 1405, 071 (2014). arXiv: 1403.5294 [hep-ex]

18. [CMS Collaboration], Note CMS-PAS-SUS-13-006

19. G. Aad et al., ATLAS Collaboration, Phys. Lett. B 718, 841 (2013). arXiv:1208.3144 [hep-ex]

20. G. Aad et al., ATLAS Collaboration, JHEP 1404, 169 (2014). arXiv: 1402.7029 [hep-ex]

21. [ATLAS Collaboration], Note ATLAS-CONF-2013-093

22. [CMS Collaboration], Note CMS-PAS-SUS-13-017

23. A. Djouadi et al., [MSSM Working Group Collaboration], The minimal supersymmetric standard model: group summary report. hep-ph/9901246

24. S.S. AbdusSalam et al., Phys. Rev. D 81, 095012 (2010). arXiv:0904.2548 [hep-ph]

25. S. Sekmen et al., JHEP 1202, 075 (2012). arXiv:1109.5119 [hep$\mathrm{ph}]$

26. A. Arbey, M. Battaglia, F. Mahmoudi, Eur. Phys. J. C 72, 1847 (2012). arXiv:1110.3726 [hep-ph]

27. A. Arbey, M. Battaglia, A. Djouadi, F. Mahmoudi, JHEP 1209, 107 (2012). arXiv:1207.1348 [hep-ph]

28. M.W. Cahill-Rowley, J.L. Hewett, A. Ismail, T.G. Rizzo, Phys. Rev. D 88(3), 035002 (2013). arXiv:1211.1981 [hep-ph]

29. A. Arbey, M. Battaglia, A. Djouadi, F. Mahmoudi, Phys. Lett. B 720, 153 (2013). arXiv:1211.4004 [hep-ph]

30. CMS Collaboration, Note CMS-PAS-SUS-12-030

31. CMS Collaboration, Note CMS-PAS-SUS-13-020

32. G.L. Kane, C.F. Kolda, L. Roszkowski, J.D. Wells, Phys. Rev. D 49, 6173 (1994). hep-ph/9312272

33. J.R. Ellis, K.A. Olive, Y. Santoso, N. J. Phys. 4, 32 (2002). arXiv:hep-ph/0202110

34. M. Drees, M.M. Nojiri, Phys. Rev. D 47, 376 (1993). arXiv:hep-ph/9207234 
35. V.D. Barger, C. Kao, Phys. Rev. D 57, 3131 (1998). arXiv:hep-ph/9704403

36. H. Baer, M. Brhlik, Phys. Rev. D 57, 567 (1998). hep-ph/9706509

37. L. Roszkowski, R. Ruiz de Austri, T. Nihei, JHEP 0108, 024 (2001). hep-ph/0106334

38. J. Ellis, K.A. Olive, Eur. Phys. J. C 72, 2005 (2012). arXiv:1202.3262 [hep-ph]

39. H. Baer, V. Barger, A. Mustafayev, JHEP 1205, 091 (2012). arXiv:1202.4038 [hep-ph]

40. B.C. Allanach, M. Battaglia, G.A. Blair, M.S. Carena, A. De Roeck, A. Dedes, A. Djouadi, D. Gerdes et al., Eur. Phys. J. C 25, 113 (2002). arXiv:hep-ph/0202233

41. M. Battaglia, A. De Roeck, J.R. Ellis, F. Gianotti, K.A. Olive, L. Pape, Eur. Phys. J. C 33, 273 (2004). hep-ph/0306219

42. S.S. AbdusSalam, B.C. Allanach, H.K. Dreiner, J. Ellis, U. Ellwanger, J. Gunion, S. Heinemeyer, M. Kraemer et al., Eur. Phys. J. C 71, 1835 (2011). arXiv:1109.3859 [hep-ph]

43. B.C. Allanach, Comput. Phys. Commun. 143, 305 (2002)

44. M. Muhlleitner, A. Djouadi, Y. Mambrini, Comput. Phys. Commun. 168, 46 (2005). hep-ph/0311167

45. A. Djouadi, M.M. Muhlleitner, M. Spira, Acta Phys. Polon. B 38, 635 (2007). hep-ph/0609292

46. A. Djouadi, J. Kalinowski, M. Spira, Comput. Phys. Commun. 108, 56 (1998)

47. F. Mahmoudi, Comput. Phys. Commun. 178, 745 (2008)

48. F. Mahmoudi, Comput. Phys. Commun. 180, 1579 (2009)

49. A. Arbey, F. Mahmoudi, Comput. Phys. Commun. 181, 1277 (2010)

50. T. Sjostrand, S. Mrenna, P.Z. Skands, JHEP 0605, 026 (2006). hep-ph/0603175

51. W. Beenakker, R. Hopker, M. Spira, hep-ph/9611232

52. S. Ovyn, X. Rouby, V. Lemaitre, arXiv:0903.2225 [hep-ph]

53. J. de Favereau et al., DELPHES 3 Collaboration, JHEP 1402, 057 (2014). arXiv:1307.6346 [hep-ex]

54. R. Aaij et al., LHCb Collaboration, Phys. Rev. Lett. 111, 101805 (2013). arXiv:1307.5024 [hep-ex]

55. S. Chatrchyan et al., CMS Collaboration, Phys. Rev. Lett. 111, 101804 (2013). arXiv:1307.5025 [hep-ex]
56. V. Khachatryan et al., [CMS and LHCb Collaborations], arXiv: 1411.4413 [hep-ex]

57. P.A.R. Ade et al., [Planck Collaboration], Astron. Astrophys. (2014). arXiv:1303.5076 [astro-ph.CO]

58. E. Aprile et al., XENON100 Collaboration, Phys. Rev. Lett. 109, 181301 (2012). arXiv:1207.5988 [astro-ph.CO]

59. D.S. Akerib et al., LUX Collaboration, Phys. Rev. Lett. 112, 091303 (2014). arXiv:1310.8214 [astro-ph.CO]

60. [ATLAS Collaboration], Note ATLAS-CONF-2013-047

61. G. Aad et al., ATLAS Collaboration, JHEP 1310, 189 (2013). arXiv:1308.2631 [hep-ex]

62. [CMS Collaboration], Note CMS-PAS-HIG-13-021

63. H. Baer, V. Barger, A. Lessa, W. Sreethawong, X. Tata, Phys. Rev. D 85, 055022 (2012). arXiv:1201.2949 [hep-ph]

64. K. Griest, D. Seckel, Phys. Rev. D 43, 3191 (1991)

65. J.R. Ellis, T. Falk, G. Ganis, K.A. Olive, M. Srednicki, Phys. Lett. B 510, 236 (2001). arXiv:hep-ph/0102098

66. M. Cacciari, G.P. Salam, G. Soyez, JHEP 04, 063 (2008), arXiv:0802.1189 [hep-ph]

67. M. Cacciari, G.P. Salam, G. Soyez, Eur. Phys. J. C 72, 1896 (2012). arXiv:1111.6097 [hep-ph]

68. A.L. Read, J. Phys. G 28, 2693 (2002)

69. T. Abe et al., [ILD Concept Group-Linear Collider Collaboration], arXiv: 1006.3396 [hep-ex]

70. H. Aihara, P. Burrows, M. Oreglia, (ed.) et al., arXiv:0911.0006 [physics.ins-det]

71. N. Alster, M. Battaglia, arXiv:1104.0523 [hep-ex]

72. S. Agostinelli et al., Nucl. Instrum. Meth. A 506, 250 (2003)

73. S. Catani, Y.L. Dokshitzer, M. Olsson, G. Turnock, B.R. Webber, Phys. Lett. B 269, 432 (1991)

74. M.A. Thomson, Nucl. Instrum. Meth. A 611, 25 (2009). arXiv:0907.3577 [physics.ins-det]

75. D.J. Jackson, Nucl. Instrum. Meth. A 388, 247 (1997)

76. D. Bailey et al., LCFI Collaboration, Nucl. Instrum. Meth. A 610, 573 (2009). arXiv:0908.3019 [physics.ins-det] 